\title{
Systematic Development of Phytophthora Species-Specific Mitochondrial Diagnostic Markers for Economically Important Members of the Genus
}

Timothy D. Miles, School of Natural Sciences, California State University Monterey Bay, Seaside, CA; Frank N. Martin, Crop Improvement and Protection Research Unit, USDA-ARS, Salinas, CA; Gregg P. Robideau and Guillaume J. Bilodeau, Canadian Food Inspection Agency (CFIA), Ottawa, Canada; and Michael D. Coffey, Department of Plant Pathology and Microbiology, University of California, Riverside

\begin{abstract}
The genus Phytophthora contains many invasive species to the U.S.A. that have the potential to cause significant damage to agriculture and native ecosystems. A genus and species-specific diagnostic assay was previously reported based on mitochondrial gene order differences that allowed for the systematic development of 14 species-specific TaqMan probes for pathogen detection (Bilodeau et al. 2014). In this study, an additional 32 species-specific TaqMan probes for detection of primarily invasive species have been validated against 145 Phytophthora taxa as well as a range of Pythium and plant DNA samples. All validated probes were found to be species-specific and could be multiplexed with a genusspecific probe. The lower limit of linear detection using purified genomic DNA ranged from 1 to $100 \mathrm{fg}$ in all assays. In addition, 124 unique TaqMan probes for Phytophthora spp. developed in silico are presented, which, if

testing confirms they are species-specific, will provide diagnostic capabilities for approximately $89 \%$ of the genus. To enhance sensitivity of detection for several species that contained a single nucleotide polymorphism (SNP) in the reverse primer, a second primer was developed that is added in a small amount to the master mix. Furthermore, a PCR-RFLP system was developed that could be used to identify individual species when multiple species are present in a sample, without requiring cloning or sequencing. Several experiments were also conducted to compare various qPCR thermal cyclers and independent validation experiments with another research laboratory to identify possible limitations when the assays are used on a range of equipment in different labs. This system represents a comprehensive, hierarchal approach to increase the detection capability and provide tools to help prevent the introduction of invasive Phytophthora species.
\end{abstract}

The genus Phytophthora is an important group of plant pathogens that are often difficult to distinguish on plant samples by symptoms alone. Several diagnostic tools have been developed that allow detection of Phytophthora species without culturing on selective media, using either immunological or molecular techniques (Martin et al. 2012). While immunological techniques are widely used, they are generally not as specific and sensitive as molecular assays and have known cross reactivity with several Pythium species (Martin et al. 2012). Traditionally, many of the molecular techniques target the internal transcribed spacer (ITS) region of the ribosomal RNA to develop markers, but other markers have been recently developed (coxl-2 spacer and Ytpl; Martin et al. 2004; Schena et al. 2008). The ability for genus-specific detection of all species that may be present and then identification with species-specific markers in a hierarchal fashion would allow for a "larger net to be cast" when examining communities where multiple similar species may be present. This is particularly important when looking at groups of organisms that make up a small percentage of environmental samples. For example, previous metagenomic research has shown that oomycetes in the rhizosphere can make up as little as $0.01 \%$ of the total microbiome while still causing damage to plants (Agler et al. 2016; Mendes et al. 2014). Assays that are currently available for detection of Phytophthora at a genus level include methods targeting the ITS region (Drenth et al. 2006) as well as the coxl-2 spacer (Martin et al. 2004), the Ytpl gene (Schena et al. 2008), and a recently reported TaqMan real time PCR

Corresponding author: Frank N. Martin; E-mail: frank.martin@ars.usda.gov

Mention of trade names or commercial products in this article is solely for the purpose of providing specific information and does not imply recommendation or endorsement by the United States Department of Agriculture (USDA).

*The $\boldsymbol{e}$-Xtra logo stands for "electronic extra" and indicates that seven supplementary tables and one supplementary alignment are available online.

Accepted for publication 27 January 2017.

This article is in the public domain and not copyrightable. It may be freely reprinted with customary crediting of the source. The American Phytopathological Society, 2017. method using the mitochondrially encoded trnM-trnP-trnM and atp9-nad9 loci (Bilodeau et al. 2014).

Bilodeau et al. (2014) described a mitochondrial marker system to detect Phytophthora species based on a gene order difference between the genus Phytophthora and the related genus Pythium, Eumycotan fungi and plants. Two loci were identified. The first locus was the trnM-trnP-trnM coding region, which was an excellent TaqMan real time PCR genus-specific marker but few sequence polymorphisms were available to develop a large number of species-specific probes (possible for $\sim 40 \%$ of species; Bilodeau et al. 2014). The second locus was larger and focused on the atp9-nad9 genes and the intervening spacer region. The amplification primers in the flanking genes produced an amplicon approximately $390 \mathrm{bp}$ in length (except $P$. bisheria and $P$. frigida, which did not amplify). A conserved genus-specific probe was placed in the atp 9 gene and putative species-specific probes were designed in the spacer region. The spacer region $(\sim 200 \mathrm{bp}$ in length, depending on the species) had significant polymorphisms among various Phytophthora species and it was possible to develop in silico probes for over $85 \%$ of Phytophthora species. Additionally, this amplification technique was found to be very sensitive; with linear detection down to 50 to $100 \mathrm{fg}$ of extracted genomic DNA (Bilodeau et al. 2014).

Sensitive and accurate detection of Phytophthora spp. is extremely important, especially from a regulatory perspective given that many species are invasive to the U.S.A. and are of economic concern. In 2009, a list of 32 invasive Phytophthora species of economic concern was developed (Schwartzburg et al. 2009), a number of which could infect a wide range of agricultural commodities and plant species in natural ecosystems. A number of new species have been described since this list was formulated, so it is likely there are additional species of regulatory concern. An updated prioritization of offshore pests was published in 2012, which included four Phytophthora spp. of regulatory concern (Crook 2012). Having the capability of fast diagnostics of these species with molecular tools in the event they were introduced would increase the chance of early detection and eradication. By targeting these species with a molecular assay that is both genus and species-specific, it might be possible to identify a wider variety of these Phytophthora spp. than with a single assay.

One drawback to the atp9-nad9 mitochondrial marker technique developed by Bilodeau et al. (2014) was that amplification for some species was optimized at different annealing temperatures and there 
were some differences in sensitivity because for a few species (including $P$. nicotinae and $P$. tentaculata) single nucleotide polymorphisms (SNPs) were present in the reverse primer annealing site (nad9). Additionally, while many species-specific probes were designed in silico, only a handful of probes were fully validated across all species (14 in total). Furthermore, when validating samples for this detection system, it was noticed that multiple species may be present in a single environmental sample (e.g., soil or irrigation water). Many researchers have reported significant diversity of Phytophthora species in various ecological systems (Hüberli et al. 2013; Jung et al. 2013; Lamour and Hu 2013); in fact, 16 different Phytophthora species were isolated in Maryland's nursery trade utilizing stream samples and baiting techniques (Bienapfl and Balci 2014). A system to identify a species that doesn't require sequencing or cloning would be helpful in these situations to reduce cost, improve efficiency, and help groups that do not have rapid access to sequencing equipment. Finally, identifying methods to transfer these assays to other researchers would be critical to ensure their increased usage and overall reliability (i.e., sensitivity and specificity).

Due to the importance of the genus Phytophthora, the atp9-nad9 marker system would be improved if more species-specific probes were validated (especially invasive species of regulatory concern), and if the user had a method for identifying the target species if multiple unknown species are present in a single environmental sample. Therefore, the goal of this study was to i) optimize amplification of the atp9-nad9 locus so that all assays can be done at a single annealing temperature, ii) validate specific probes for species of regulatory importance, iii) develop a post-PCR method with the atp9-nad9 marker system to identify individual Phytophthora species when multiple species are present, and iv) validate these markers in a separate lab and develop a protocol for transferring the technology to other users.

\section{Materials and Methods}

Phytophthora, Pythium, Phytopythium, and plant species used. A total of 225 Phytophthora isolates were used in this study representing 145 taxa (Supplementary Table S1). These isolates included three different subspecies of P. alni (alni, multiformis, and uniformis), three phylogenetic groups of P. cryptogea (GI, GII, and sp. kelmania GIII), six phylogenetically distinct species (sp. aff. brassicae-1, sp. aff. brassicae-2, sp. aff. colocasiae-1, sp. aff. erythroseptica, sp. aff. siskiyouensis, cinnamomi var robiniae, and citricola clade $\mathrm{E})$, and two naturally occurring hybrids $(P . \times$ pelgrandis and $P . \times$ serendipita) (Jung and Burgess 2009; Martin et al. 2014). All Phytophthora isolates are available from the World Phytophthora Genetic Resource Collection at the University of California, Riverside (http://phytophthora.ucr.edu/databasemain.html). The assay described in this manuscript was updated from Bilodeau et al. (2014) in order to confirm specificity with new amplification conditions. To check the specificity of this assay, 21 Pythium spp., one Phytopythium sp., and six plant species were retested (Supplementary Table S2). Additional information about the Pythium and Phytopythium isolates can be found in Martin et al. (2004).

Sequence alignments of mitochondrial loci used for targets were constructed using the software Geneious v.4.7.6 (Biomatters Ltd., Auckland, New Zealand), and included the trnM-trnP-trnM, and atp9-nad9 regions present in Phytophthora species, and the coxl region in plants (for development of a plant internal control). The total number of sequences used to develop the marker system (representing approximately 145 Phytophthora taxa) was 633 (including multiple isolates of the same species to assess intraspecific variation in the target) for the atp9-nad9 region. Additional information on how these sequences were obtained can be found in Bilodeau et al. (2014). Primers in this study were primarily designed manually based on alignments; however, melting temperature and $\mathrm{GC} \%$ was calculated using the OligoAnalyzer Tool 3.1 (Integrated DNA Technologies, Coralville, IA) (Table 1).

DNA samples of each Phytophthora isolate were obtained from the World Phytophthora Collection at a concentration of $10 \mathrm{ng} / \mu \mathrm{l}$. This concentration was verified periodically using a Nanodrop 2000 instrument (Thermo Fisher Scientific, Waltham, MA). For validating species-specific probes, four 96-well master plates were made by diluting this DNA to a concentration of $1 \mathrm{ng} / \mu \mathrm{l}$. However, for all standard curve plots and limit of detection data provided in this study, a Qubit Fluorometer and the Quant-iT dsDNA HS Assay Kit (Invitrogen, Carlsbad, CA) was utilized following the manufacturer's instructions to more precisely determine DNA concentrations using $10 \mu 1$ of $1 \mathrm{ng} / \mu \mathrm{l}$ DNA (based on initial Nanodrop values).

In silico probe design for the atp9-nad9 locus. A database of 124 Phytophthora in silico species-specific probes was designed for the atp9-nad9 mitochondrial locus and several of these probes were selected for validation in this study based on perceived use and feasibility (e.g., higher GC content and polymorphic probe binding regions) (Supplementary Table S3). Specific attention was paid to Phytophthora species considered to be invasive to the United States (Crook 2012; Schwartzburg et al. 2009) (Supplementary Table S4). Probes were designed manually using alignment software Geneious 4.7.6. Probes were also checked for GC\%, melting temperature, and primer/probe incompatibility using the OligoAnalyzer Tool 3.1. All probes selected for validation were ordered using a variety of dual labeled fluorometric markers from LGC Biosearch Technologies (Novato, CA). Species-specific probes were labeled at the $5^{\prime}$ end with either a HEX or Quasar 670 dye in order to be multiplexed with the Phytophthora genus-specific probe (labeled with FAM) and plant internal control probe (CAL Fluor Red 610 dye) (Table 2).

Optimization of TaqMan amplification. In an effort to enhance sensitivity of amplification for some species and allow all assays to be run at a single annealing temperature, prior to validating all species-specific probes a "Bandaid" primer was designed to be added to the atp9-nad9 assay described in Bilodeau et al. (2014). The purpose of this extra reverse primer was to allow primers to bind when a SNP was present in the reverse primer-binding site (Fig. 1). A variety of Bandaid primer concentrations were tested from $500 \mathrm{nM}$ down to $1 \mathrm{nM}$. Following these optimization experiments, $8 \mathrm{nM}$ was chosen as the appropriate final concentration. With the addition of this Bandaid primer, the standard atp9-nad9 amplification conditions were modified from Bilodeau et al. (2014) as follows: PhyG_ATP9_ 2FTail and PhyG-R6_Tail (500 nM each), Bandaid primer (8 nM), ATP9_PhyG2_probeR and desired species-specific probe $(50 \mathrm{nM}$ each), FMPI2b and FMPI3b (12.5 nM each), Plant CAL-Red probe (10 nM), 5 Prime Master Mix (catalog number 2200100, 5 Prime Inc., Gaithersburg, MD), and 6 additional $\mu \mathrm{l}$ of $25 \mathrm{mM} \mathrm{Mg}$ in a $25 \mu \mathrm{l}$ reaction (Supplementary Table S5). The plant primers and probe were always included in all experiments because the ultimate goal of these assays was to detect Phytophthora from environmental samples and the plant control was used to assess if the DNA was amplifiable. Previous research had different annealing temperatures for various species due to the presence of SNPs; because of this, three species were used to test for similar sensitivity and specificities across various genera: P. nicotianae (P1452), P. ramorum (Prg-2), and $P$. tentaculata (P8497). Purified DNA was loaded separately into a reaction at a concentration ranging from $300 \mathrm{pg}$ to $3 \mathrm{fg}$ (based on Qubit Fluorometer values). Standard curve plots of the log of concentration and cycle threshold values were plotted based on two replicate reactions from each species. Since the fundamental reaction components had changed slightly from Bilodeau et al. (2014), the 14 species-specific probes from this previous study were revalidated. Following these optimization experiments all future amplifications were performed using the following thermal cycling conditions: an initial $2 \mathrm{~min}$ at $95^{\circ} \mathrm{C}$, followed by 50 cycles of $95^{\circ} \mathrm{C}$ for $15 \mathrm{~s}$ and $57^{\circ} \mathrm{C}$ for $1.5 \mathrm{~min}$ with fluorometric data being collected at the end of each cycle. In addition to validating annealing temperature, two master mixes were tested side by side, using a serial dilution of P. ramorum (Prg-2) DNA, namely the 5 Prime Master Mix as described above and the PerfeCTa qPCR ToughMix (Quanta Biosciences, Gaithersburg, MD). The primary reason this was tested was the 5 Prime Master mix is no longer being produced on a commercial scale (as of 2016) and an alternative master mix will be required to utilize the atp9-nad9 assay outlined in this manuscript under these conditions.

Testing for sensitivity and specificity. To test for specificity and sensitivity, purified DNA from each of the 225 Phytophthora isolates $(1 \mathrm{ng} / \mu \mathrm{l})$ was maintained in four $96-$ well master plates. The master 
Table 1. Amplification primers, Phytophthora genus-specific and plant-specific qPCR probes, and sequencing primers utilized in this study

\begin{tabular}{|c|c|c|c|c|}
\hline Name & Sequence $5^{\prime}-3^{\prime}$ (with modification if appropriate) ${ }^{\mathbf{a}}$ & $\begin{array}{l}\text { Calculated } \\
\operatorname{Tm}\left({ }^{\circ} \mathbf{C}\right)^{\mathbf{b}}\end{array}$ & Target & Originated from \\
\hline \multicolumn{5}{|l|}{ Amplification primers } \\
\hline PhyG_ATP9_2FTail & AАTAАATCATAАССТTСТTTACAACAAGAATTAATG & 54.2 & atp 9 & Bilodeau et al. 2014 \\
\hline PhyG-R6_Tail & AАTAАATCATAАATACATAАTTCATTTTTATA & 47.1 & $\operatorname{nad} 9$ & Bilodeau et al. 2014 \\
\hline Bandaid primer & AATAAATCATAAATACATAATTCRTTTTTRTA & 48.7 & $\operatorname{nad} 9$ & This study \\
\hline FMPI2b & GCGTGGACCTGGAATGACTA & 56.8 & Plant $c o x$ & Bilodeau et al. 2014 \\
\hline FMPl3b & AGGTTGTATTAAAGTTTCGATCG & 51.0 & Plant cox & Bilodeau et al. 2014 \\
\hline \multicolumn{5}{|l|}{$q P C R$ probes } \\
\hline ATP9_PhyG2_probeR & [Fam]AAAGCCATCATTAAACARAATAAAGC [BHQ1] & 52.2 & atp9 & Bilodeau et al. 2014 \\
\hline Plant CAL-Red probe & [CALFluorRed610]CTTTTATTATCACTTCCGGTACTGGCAGG [BHQ2] & 58.8 & Plant cox & Bilodeau et al. 2014 \\
\hline \multicolumn{5}{|c|}{ Nested sequencing primers } \\
\hline Nad9-F & TACAACAAGAATTAATGAGAAC & 46.4 & atp 9 & Bilodeau et al. 2014 \\
\hline Nad9_Rseq1 & GTAAAATTTGTAATAAATATTGACT & 44.4 & $\operatorname{nad} 9$ & This study \\
\hline
\end{tabular}

${ }^{a}$ Extra bases on the $5^{\prime}$ end of primers are denoted in bold and fluorophores and quenchers are denoted with brackets.

b Primer or probe melting temperature was calculated using the OligoAnalyzer 3.1 (Integrated DNA Technologies, Coralville, IA).

Table 2. Comprehensive list of species-specific probes that have been developed and validated for the atp9-nad9 locus (including those from Bilodeau et al. 2014)

\begin{tabular}{|c|c|c|c|c|c|c|}
\hline Probe name & Phytophthora spp. & Probe sequence $\left(5^{\prime}-3^{\prime}\right)$ & Modification & $\begin{array}{l}\text { Main } \\
\text { isolate }\end{array}$ & $\begin{array}{c}\text { Limit of } \\
\text { detection }\end{array}$ & Slope \\
\hline Palni_nad9sp_probe1 & alni & AATAGATATATACGTATATTTAACGCATAATTAGC ${ }^{\mathrm{a}}$ & Quasar670/BHQ2 & P16203 & $10 \mathrm{fg}$ & $y=-2.996 x+22.006$ \\
\hline Paustrocedrae_n9_prb & austrocedrae & TATACAGTGTTACGTATGTACTTTAGGGTAATTT ${ }^{b}$ & Hex/BHQ1 & P15132 & $100 \mathrm{fg}$ & $y=-3.358 x+18.5914$ \\
\hline Pboehmeriae_n9_prb & boehmeriae & ACCTGTTTAATATAATAAAAATTATTTTAAT ${ }^{\mathrm{b}}$ & Quasar670/BHQ2 & P6950 & $10 \mathrm{fg}$ & $y=-3.1991 x+18.7656$ \\
\hline Pbotryosa_n9_prb & botryosa & TGTTATGTGTATACTRTTATATAAAATTTATAT ${ }^{b}$ & Hex/BHQ1 & P3425 & $10 \mathrm{fg}$ & $y=-3.2765 x+16.8073$ \\
\hline Pcact_nad9sp_probe2 & cactorum & TTACATGTTATATAATTATTAACACTATTTATAAAA ${ }^{\mathrm{a}}$ & Quasar670/BHQ2 & P0714 & $10 \mathrm{fg}$ & $y=-3.14 x+20.94$ \\
\hline Pcajani_n9_prb & cajani & ACTACGTATATTTTTGATATATGTATACCTATACA & Hex/BHQ1 & P3105 & $100 \mathrm{fg}$ & $y=-3.365 x+19.7649$ \\
\hline Pcambi_nad9sp_probe1 & cambivora & ATCCTATAATAGGTATATATGTACATTTAATGCA ${ }^{\mathrm{a}}$ & Hex/BHQ1 & P0592 & $100 \mathrm{fg}$ & $y=-3.34 x+22.973$ \\
\hline Pcaptiosa_nad9sp_probe & captiosa & ATAAAATATATAAATACTGCAGTAAAATTATAATA ${ }^{\mathrm{b}}$ & Hex/BHQ1 & P10719 & $10 \mathrm{fg}$ & $y=-3.74 x+21.973$ \\
\hline Pcinn_nad9sp_probe1 & cinnamomi & AAGAAATATTTAGTTTATTAATATATAATATAACT & Quasar670/BHQ2 & P2110 & $100 \mathrm{fg}$ & $y=-3.063 x+20.498$ \\
\hline PcitSS_probe & citricola & CAGGTTATATAACTACTGATATTAGGAATTAAACT ${ }^{\mathrm{b}}$ & Quasar670/BHQ2 & 0716 & $10 \mathrm{fg}$ & $y=-3.4631 x+18.5254$ \\
\hline Pcit_nad9sp_T1F & citricola group & AATAATAGTTTATTTTTTTGATATATAAATATTTAT & CALFluorRed610/BHQ2 & P0716 & $10 \mathrm{fg}$ & $y=-3.043 x+22.058$ \\
\hline Pcland_nad9sp_probe & clandestina & ATATAAATTTTTATTATATTTTATATAACTGTTA & Hex/BHQ1 & P3942 & $10 \mathrm{fg}$ & $y=-3.133+19.4095$ \\
\hline Peryth_nad9sp_probe & erythroseptic & TCGGTACTAATGCGATAATCTATCCTATTTTTAG & Hex/BHQ1 & P1699 & $100 \mathrm{fg}$ & $y=-3.4524+18.9415$ \\
\hline Pfol_nad9sp_probe2 & foliorum & ATATTTATACACAAAGGTAAAAATACATTAT ${ }^{b}$ & Quasar670/BHQ2 & P10969 & $100 \mathrm{fg}$ & $y=-3.306 x+18.8912$ \\
\hline PfraVf_nad9sp_TaqMan2 & fragariae & ATCTCGTAATAGATATATATGTATATTTAATACGT & Hex/BHQ1 & P3821 & $10 \mathrm{fg}$ & $y=-3.21 x+19.52$ \\
\hline Pidaei_nad9sp_probe & idaei & СATAATTATTAATACTGTTTATAAAAATGTTTTAT & Hex/BHQ1 & P6767 & $10 \mathrm{fg}$ & $y=-3.5497+21.6181$ \\
\hline Pinfest_n9_prbc & infestans & TTAYTYTGTTACGTAATTATTTATAGTAAATATC & Quasar670/BHQ2 & P10650 & $10 \mathrm{fg}$ & $y=-3.4554+19.9819$ \\
\hline Pkatsurae_n9_prb & katsurae & TGTTATATAAGTATTWAATTRAAAATRTATAAATT ${ }^{\mathrm{b}}$ & Quasar670/BHQ2 & 187 & $100 \mathrm{fg}$ & $y=-3.1434+21.3003$ \\
\hline Pker_nad9sp_1Fb & kernoviae & TTATATTATTCACAGATTTATTAATTTTTTTCTA ${ }^{\mathrm{a}}$ & Quasar670/BHQ2 & P10681 & $100 \mathrm{fg}$ & $y=-3.1100 x+21.91$ \\
\hline Pgona_nad9sp_probe2 & lacustris & ATAATACACGTATACTTAAACCCTTTTTAGTA ${ }^{\mathrm{a}}$ & Quasar670/BHQ2 & P10337 & $10 \mathrm{fg}$ & $y=-3.546 x+17.936$ \\
\hline Plat_nad9sp_probe1 & lateralis & ACGTCTGCACTGAAAGACGTATAAAAT ${ }^{b}$ & Quasar670/BHQ2 & P3888 & $10 \mathrm{fg}$ & $y=-3.6392 x+20.7275$ \\
\hline Pmacrochl_n9_prb ${ }^{\mathrm{d}}$ & macrochla & AWTATAATTATTAAATAATAAATCACCTTAATTTAAT ${ }^{\mathrm{b}}$ & Quasar670/BHQ2 & P10267 & $10 \mathrm{fg}$ & $y=-3.3257 x+20.1772$ \\
\hline P meadiinew1 & meadii & TTTATGTTATGTGCATACTATTATATAATATT ${ }^{\mathrm{b}}$ & Quasar670/BHQ2 & P6128 & $10 \mathrm{fg}$ & $y=-3.4685 x+18.8229$ \\
\hline Pmegaka_n9_prb & megakarya & TTTAATGTTATATAAATACTTATATTAAAATATAT & Quasar670/BHQ2 & P8516 & $100 \mathrm{fg}$ & $y=-3.1487 x+21.8835$ \\
\hline Pmelonis_n9_prb & melonis & TACATATATTTGATATATAAATACCGTTACGTAT & Hex/BHQ1 & P10994 & $10 \mathrm{fg}$ & $y=-3.3615 x+18.4824$ \\
\hline Pmeng2 & mengei & TATTTATTTTAATTATATATATACTGGTAAATAAA ${ }^{\mathrm{b}}$ & ex/BHQ1 & 273 & $10 \mathrm{fg}$ & $y=-3.4375 x+18.1877$ \\
\hline Pmultive_n9_prb & multivesiculata & AGGAATATATAGTTACTGTTAACTAAAAATAAAAT ${ }^{\mathrm{b}}$ & Quasar670/BHQ2 & P10410 & $10 \mathrm{fg}$ & $y=-3.3724 x+19.0234$ \\
\hline Pmult_probe2 2 & multivora & ATATTAATGAAAATTTTCGTTAATATATTTTATTT ${ }^{b}$ & Quasar670/BHQ2 & P7902 & $10 \mathrm{fg}$ & $y=-3.4631 x+19.6584$ \\
\hline Pnicot_ATP9_Probe1 & nicotianae & ATGTTATATCATTATTTTTTATTATATATACAAAT & BHQ2 & 1452 & $100 \mathrm{fg}$ & $y=-3.316 x+21.956$ \\
\hline Ppalm_nad9sp_probe2 & palmivora & TATAATTACTTAGRCYTGAGTATTTAAATTGAAA ${ }^{\mathrm{a}}$ & Quasar670/BHQ2 & P0255 & $10 \mathrm{fg}$ & $y=-3.252 x+19.657$ \\
\hline Pparvispora_n9_prb & parvispora & AATGRTATATAYRTACTTTTTWAAAAAACTCGA ${ }^{\mathrm{b}}$ & Hex/BHQ1 & P8495 & $100 \mathrm{fg}$ & $y=-3.6103 x+19.0857$ \\
\hline Ppinifolia_n9_prb & pinifolia & AAGGTGTTATACGTATACTTAACCCCTTTTAG ${ }^{\mathrm{b}}$ & Hex/BHQ1 & P16100 & $1 \mathrm{fg}$ & $y=-3.6169 x+15.7877$ \\
\hline Ppistaciae_n9_prb & pistaciae & TACTACACATTTGATATATAAATACAAATATACT ${ }^{\mathrm{b}}$ & Quasar670/BHQ2 & P6197 & $10 \mathrm{fg}$ & $y=-3.2813 x+19.6132$ \\
\hline Ppluri_probel 1 & plurivora & AGGTTATATACTTACTGATACTGAAAATTAAATTA ${ }^{\text {b }}$ & Hex/BHQ1 & P10679 & $100 \mathrm{fg}$ & $y=-3.4419 x+19.4267$ \\
\hline Pporrinew1 & porri & AAATTTTATTATAAAGTTATATGTGTACTTT $\mathrm{b}$ & Quasar670/BHQ2 & P7518 & $10 \mathrm{fg}$ & $y=-3.2625 x+20.5183$ \\
\hline Pprimul_n9_prb & primulae & ATTTTATTATGAAGTCACATGTGTACTTTATAAA & Hex/BHQ1 & P10333 & $10 \mathrm{fg}$ & $y=-3.4451 x+19.6724$ \\
\hline Ppssyr_ATP9_Pro & pseudosyr & TTAGATATGTAAGTACTTATAGTGTTTATATT ${ }^{\mathrm{a}}$ & Quasar670/BH & & $10 \mathrm{fg}$ & $y=-3.362 x+20.457$ \\
\hline Pquercina_nad9sp_probe1 & quercina & ATTATATTCTTATGTTATATAAYCACTAATACTG & Hex/BHQ1 & P10334 & $1 \mathrm{fg}$ & $y=-3.4109 x+18.7852$ \\
\hline Pquininea_nad9sp_probe ${ }^{\mathrm{d}}$ & quininea & ATCACCTTAATTTAATATATTCCTATTTTAATAAT & & P3247 & $100 \mathrm{fg}$ & $y=-3.5767 x+19.7596$ \\
\hline Pram_nad9sp_1F & ramorum & ACGTTACGTCTAGACTTGTATTATGCATTG ${ }^{\mathrm{a}}$ & & P10301 & $10 \mathrm{fg}$ & $y=-3.2100 x+21.56$ \\
\hline PfraVrubi_Atp9_TaqMan1 & rubi & ATATATACGTGTATTTAATGCATAATCAGCTA & Quasar670/BHQ2 & P3289 & $10 \mathrm{fg}$ & $y=-3.1631 x+17.334$ \\
\hline Psyr_nad9sp_probe1 & syringae & TACTTTTARCTAAATGTTAACTATTTTTCTAA ${ }^{\mathrm{a}}$ & Quasar670/BHQ2 & P10330 & $10 \mathrm{fg}$ & $y=-2.952 x+21.067$ \\
\hline Ptentac_n9_prb & tentaculata & TTATATATTTGTTATATAATTATTTATAATAACTA & Quasar670/BHQ2 & P8497 & $100 \mathrm{fg}$ & $y=-3.1178 x+23.9157$ \\
\hline Pvignae_n9_prb & vignae & ACTACATATACTTTTGATATACATATACCTATACA & Hex/BHQ1 & P3019 & $10 \mathrm{fg}$ & $y=-3.5133+19.2176$ \\
\hline
\end{tabular}

a Denotes probes that were developed and validated in Bilodeau et al. (2014).

${ }^{\mathrm{b}}$ Denotes probes that were developed and validated in this manuscript.

${ }^{\mathrm{c}}$ The P. infestans probe (Pinfest_n9_prb) was found to cross react with several members of the Phytophthora genus that are in clade 1c (including P. andina, P. mirabilis and P. ipomoeae but not P. pistaciae).

d Probes for P. macrochlamydospora (Pmacrochl_n9_prb) and P. quininea (Pquininea_nad9sp_probe) were found to be unable to be resolved from each other. 
mix was made and template was added from each master plate using an 8-well multichannel pipette. The assays were developed and tested using the CFX 96 Touch machine (Bio-Rad Laboratories, Hercules, CA). Two species-specific probes were tested in the same reaction, meaning each probe was always in the presence of another speciesspecific probe (with a different fluorophore) in order to decrease cost. If an unexpected amplification was observed, the assay was repeated at least twice; once a probe was considered specific using the BioRad CFX 96 Touch, it was sent to the partnering laboratory (Ottawa, Canada) and tested on a different qPCR thermal cycler (ABI viiA7, Applied Biosystems, Carlsbad, CA). Each of the 46 species-specific probes listed in Table 2 were tested for sensitivity using a serial dilution from $1 \mathrm{ng}$ to $1 \mathrm{fg}$ of DNA from the reference isolate for that species (Table 2). A standard curve plot was plotted from this dilution series and summarized in Table 2. Efficiency of the standard curve plot was calculated using the QPCR standard curve slope to efficiency calculator (http://www.genomics.agilent.com/ biocalculators/calcSlopeEfficiency.jsp). Additionally, the limit of detection was determined for each species using this DNA. The efficiency can also be calculated using the slope info with the equation $\mathrm{E}=\left(10^{(-1 / \text { slope })}-1\right) \times 100$.

Restriction fragment length polymorphism (RFLP) for species identification. For all 145 Phytophthora taxa, the atp9-nad9 region in silico amplicon sizes and restriction digests were predicted for the enzymes AseI and DraI in an effort to develop a PCR-RFLP system (Supplementary Table S7). To test in silico predictions, the atp9-nad9 region of a subset of species was amplified utilizing the qPCR protocol described above and PCR products separated using a 1.5\% TBE agarose gel to confirm amplification and estimate DNA concentration. Amplicons were individually digested with each enzyme following instructions of the manufacturer (New England Biolabs, Ipswich, MA). These

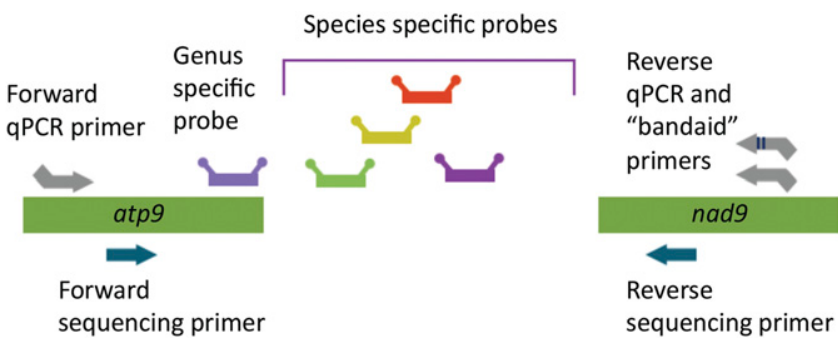

Fig. 1. Graphical representation of the Phytophthora TaqMan detection system for the mitochondrial locus atp9-nad9, originally by Bilodeau et al. (2014) and further refined in this study. fragments were then run on a 3\% TBA gel (Lonza group, Basel, Switzerland) with a 25 b ladder (New England Biolabs) and compared with each other to determine if individual species could be distinguished. To identify specific species using this PCR-RFLP method, a size difference of at least $10 \mathrm{bp}$ or larger for bands $100 \mathrm{bp}$ or greater is needed and both digestions were required.

Detection devices and independent validations. The assays were optimized utilizing the CFX 96 Touch qPCR thermal cycler; however, all assays were further validated using an ABI viiA7 unit in Ottawa, Canada, by different personnel. Once both laboratories considered all probes to be specific, we conducted a series of experiments to transfer the assay to other laboratories. A protocol was then devised to transfer technology to future laboratories using three progressive steps to confirm amplification of test samples. The first step involved sending positive controls and utilized the standard amplification protocol with the addition of species-specific probes for $P$. fragariae (fraVf_nad9sp_TaqMan2) and P.rubi (PfraVrubi_Atp9_TaqMan1). This step included four reactions replicated three times and included the reactions: 1) $P$. fragariae $(0.5 \mathrm{ng}$, isolate P3821), 2) $P$. rubi (0.5 ng, isolate P3289), 3) extracted strawberry DNA (5 ng) spiked with a dilute amount of $P$. fragariae $0.05 \mathrm{ng}$, and 4 ) water. Once this step was passed, users were expected to change their species-specific probe in their master mix to only P. ramorum for steps 2 and 3 (Pram nad9sp_1F). The second step included a serial dilution that used purified DNA from $P$. ramorum (isolate P10301), which began at a concentration of $0.5 \mathrm{ng}$ and was diluted down to $5 \mathrm{fg}$ and included three replicate reactions in order to investigate the limit of detection and overall slope on the standard curve plot. The third and final step included 20 samples sent blind described in Table 3 from various Phytophthora infected plant extracts, uninfected plant extracts, purified Phytophthora, and Pythium DNA samples. These reactions were repeated in triplicate to test for problems with specificity. These technology transfer experiments were performed on three different thermal cyclers at two different locations: ABI viiA7 (Applied Biosystems), CFX 96 Touch (Bio-Rad), and Lightcycler 480 (Roche), to evaluate how well the assay worked when different equipment was used.

\section{Results}

A TaqMan system for detection of Phytophthora species at genus and species-specific level. The Phytophthora atp9-nad9 mitochondrial marker system was optimized and revalidated, building on the detection system described in Bilodeau et al. (2014). Like previous research from Bilodeau et al. (2014), the 5 Prime Master Mix was found to be critical in these experiments as it had lower $C_{t}$ than other master mixes and recent research has shown that PerfeCTa

Table 3. Blind samples used in technology transfer experiments to test for specificity of the atp9-nad 9 assay developed in the study to detect Phytophthora species at a genus and species-specific level

\begin{tabular}{|c|c|c|c|c|}
\hline Sample number & Description & Sample type & Genus positive & P. ramorum positive \\
\hline 1 & Rhamnus californica infected with $P$. cactorum & Field sample & + & - \\
\hline 2 & Citrus sp. infected with $P$. citrophthora & Field sample & + & - \\
\hline 3 & Rubus sp. infected with $P$. rubi & Field sample & + & - \\
\hline 4 & P. ramorum & Phytophthora purified DNA & + & + \\
\hline 5 & Fragaria $\times$ ananassa & Field sample & - & - \\
\hline 6 & Rubus sp. & Field sample & - & - \\
\hline 7 & Pythium paroecandrum & Non-Phytophthora purified DNA & - & - \\
\hline 8 & Pythium irregulare & Non-Phytophthora purified DNA & - & - \\
\hline 9 & Pythium ultimum & Non-Phytophthora purified DNA & - & - \\
\hline 10 & Fusarium oxysporum & Non-Phytophthora purified DNA & - & - \\
\hline 11 & P. kernoviae & Phytophthora purified DNA & + & - \\
\hline 12 & Citrus sp. & Non-Phytophthora purified DNA & - & - \\
\hline 13 & Rubus sp. & Field sample & - & - \\
\hline 14 & P. lateralis & Phytophthora purified DNA & + & - \\
\hline 15 & P. foliorum & Phytophthora purified DNA & + & - \\
\hline 16 & Umbellularia californica & Field sample & - & - \\
\hline 17 & Umbellularia californica & Field sample & - & - \\
\hline 18 & Umbellularia californica infected with $P$. ramorum & Field sample & + & + \\
\hline 19 & P. ramorum & Phytophthora purified DNA & + & + \\
\hline 20 & Water & N/A & - & - \\
\hline
\end{tabular}


qPCR ToughMix (Quanta Biosciences) also worked equally in amplification assays for the atp9-nad 9 system and had similar detection limits for P. ramorum and PCR amplification efficiency (data not shown). A set of sequencing primers were developed to confirm a PCR positive from atp9-nad9 amplicons, namely Nad9_Rseq1 (this study) and Nad9-F (Bilodeau et al. 2014), which target the nad9 and atp9 genes, respectively (Fig. 1, Table 1). Confirmation of a positive atp9-nad9 product was successfully conducted on a subset of amplifications from 20 different Phytophthora spp. and the resulting sequences
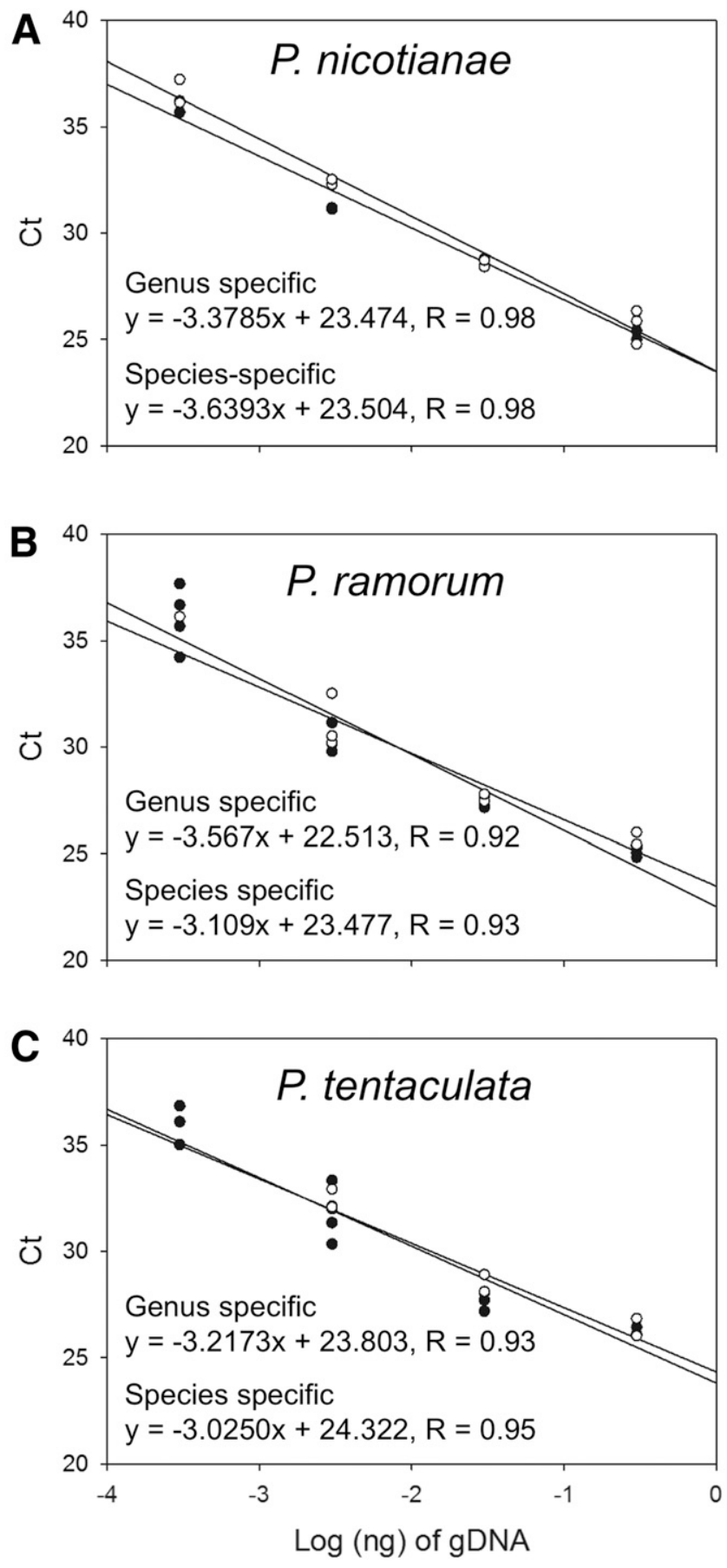

\section{Genus specific $\bigcirc$ Species specific $O$}

Fig. 2. Optimization of amplification using the "Bandaid" primer to increase the amplification sensitivity of five species, which contain a SNP in the reverse nad9 primer. Standard curve plots of sensitivity for three important Phytophthora species detected using the atp9-nad9 mitochondrial locus with genus (closed circles) and species-specific (open circles) probes. A, P. nicotianae. B, P. ramorum, and C, $P$. tentaculata. All amplifications were performed at the same annealing temperature. were compared with a local BLAST database provided in Bilodeau et al. (2014, Supplemental Alignment) confirmed species identification.

An in silico database was created for potential probes for the atp9nad 9 region and a total of 124 probes were designed with various levels of specificity. In total, 46 species-specific probes were fully validated against master plates and all probes identified, with a few exceptions, were specific when tested against other Phytophthora and Pythium spp. The probes for P. macrochlamydospora (Pmacrochl_n9_prb) and $P$. quininea (Pquininea_nad9sp_probe) were found to be unable to differentiate between these species. Likewise, a probe for P. botryosa (Pbotryosa_n9_prb) was found to cross react with $P$. meadii but was otherwise specific. Also, the $P$. infestans probe (Pinfest_n9_prb) was found to cross react with several members of the Phytophthora genus that are in clade 1c (including $P$. andina, $P$. mirabilis, and $P$. ipomoeae, but not $P$. phaseoli).

Fluorophore modifications to the $5^{\prime}$ ends of probes (either Hex or Quasar 670) had no effect on detection and appear to be interchangeable (data not shown). The limit of detection across all 46 probes varied using purified DNA of targeted species from $1 \mathrm{fg}$ to $100 \mathrm{fg}$ using a representative isolate for each species (Table 2). Standard curve plot slopes for all the 46 species-specific probes (using the log of DNA concentration and $C_{t}$ value) were generally between -3.74 and -2.95 , which correlates to qPCR efficiencies of 85 to $118 \%$.

Optimizing the atp9-nad9 detection system for all Phytophthora spp. for similar levels of sensitivity. Prior to testing species-specific probes, an additional reverse amplification primer (Bandaid primer) was added to the atp9-nad9 assay from Bilodeau et al. (2014) since seven known species had SNPs in nad9 reverse primer binding site (P. alticola, $P$. cryptogea $[\mathrm{GII}]$, P. elongata, $P$. nicotinae, $P$. medicaginis, $P$. tentaculata, and $P . \times$ pelgrandis). These SNPs caused assays in Bilodeau et al. (2014) to be optimized at different annealing temperatures (particularly with $P$. nicotinae). This Bandaid primer was developed in order to standardize annealing temperatures across members of the Phytophthora genus at a fixed $57^{\circ} \mathrm{C}$ (Fig. 1, Table 1). A variety of primer concentrations were tested and at high concentrations, the Bandaid primer appeared to limit assay sensitivity, causing a reduced limit of detection when present at concentrations higher than $25 \mathrm{nM}$ (data not shown). It was determined that a concentration of $8 \mathrm{nM}$ of Bandaid primer significantly increased sensitivity of Phytophthora spp. that contained SNPs in the reverse primer binding sites without sacrificing overall detection sensitivity. Using this new assay, a serial dilution series of purified DNA from $P$. nicotinae, $P$. ramorum, and $P$. tentaculata was tested, qPCR amplification efficiencies of 97.6, 90.6, and $104.4 \%$, respectively, were observed. The limit of detection in all of these experiments was $500 \mathrm{fg}$ across all three species (Fig. 2).

Technology transfer of this detection system to various qPCR machines in multiple laboratories. A process was developed in order to validate results on different qPCR platforms and to transfer this technology to another research or diagnostic laboratory. Three different qPCR thermal cyclers (CFX 96 Touch, LightCycler 480, and ABI viiA7) were tested and a three-step process developed. The first step involved performing amplifications using three DNA samples resulted in similar amplifications across all three platforms. Template concentrations in these samples were $5 \mathrm{ng}$ for $P$. fragariae or $P$. rubi, resulting in $C_{t}$ values between 16.46 and 18.11 (depending on which probe or machine was utilized) (Table 4). Also in this step, a strawberry sample spiked with $0.05 \mathrm{ng}$ of $P$. fragariae DNA produced similar results with $\mathrm{C}_{\mathrm{t}}$ values for Phytophthora spp. ranging from 20.20 to 21.60, depending on which machine was utilized, with detection occurring earlier in the CFX 96 Touch. Optically, on all machines, there was no bleedthrough observed from one channel to another during the amplification.

The second step used a serial dilution of purified $P$. ramorum DNA to compare sensitivity across all the machine platforms. Similar results were observed in all machines, with slope values ranging from -3.396 to -3.158 and amplification efficiencies of 97 to $107 \%$, respectively. The limit of detection in all of these machines was 500 fg regardless of whether genus or species-specific probes were used for detection (Table 4, Fig. 3). For the third step, a group of 20 samples sent blind was used to confirm similar detection results across each machine. Identical levels of detection were observed for the 
Phytophthora purified DNA, field samples, and non-Phytophthora purified DNA samples on all three machines (Table 4).

Two machines were located in one research laboratory (CFX 96 Touch and Lightcycler 480) and the ABI viiA7 was located in another location with different personnel performing the amplifications. In general, results were similar for specificity (step 1), amplification efficiency and limit of detection (step 2), and consistent detection on a variety of samples (step 3) (Table 4). Periodically, the cycle threshold line needed to be adjusted on the ABI viiA7 to avoid noise. Finally, the Lightcycler 480 was unable to detect the CalFlorRed 610 dye in the internal plant control due to the lack of the appropriate filter.

Analyzing atp9-nad9 PCR amplicons using restriction fragment length polymorphisms (RFLP). A system to evaluate atp9nad9 amplicons following PCR was developed using RFLP analysis. The AT rich regions common in the atp9-nad9 spacer region made it possible to target this region with two restriction enzymes that cut in AT rich sites, AseI and DraI. A subset of 11 Phytophthora species were amplified with amplicon sizes of approximately $390 \mathrm{bp}$ (except $P$. gonapodyides, which produced an amplicon approximately 494 bp, Fig. 4). Following amplification, each restriction enzyme was used individually to digest products and these fragments were visualized by gel electrophoresis. Under certain circumstances, such as differentiating two species from a specific host, this method was highly effective at identifying the species present (e.g., differentiating $P$. cinnamomi from $P$. menge $i$ when working with avocado root rot). By using a combination of results from both AseI and DraI digestion, it was predicted that, based on banding pattern alone, 36 species could be independently identified when compared with all members of the Phytophthora genus (Table 5), meaning that these species had fragments $>100 \mathrm{bp}$ and differed in size by at least $10 \mathrm{bp}$ or had different fragment patterns using both enzymes. While it was not possible to differentiate all species evaluated by gel electrophoresis, for many, the restriction patterns obtained at least narrowed down the number of species that might be present, particularly if the user knew something about which species to expect. For example, if a user was studying avocado root rot (caused by $P$. cinnamomi or
P. mengei), it was quite easy to differentiate the species using this method (Fig. 4). The majority of species in clades 6 to 10 are more divergent in the atp9-nad9 spacer region and could potentially be easier to identify by PCR-RFLP analysis compared with clades 1 to 5 . Furthermore, for some species, a specific restriction enzyme could be utilized for species identification to differentiate them from all other members of the genus. For example, $P$. captiosa, $P$. gonapodyides, and $P$. ramorum could potentially be identified using $P$ st $I$, EcoRV, or XbaI, respectively.

\section{Discussion}

Having a molecular detection assay for Phytophthora that generates a single amplicon that can be used for both genus and species-specific

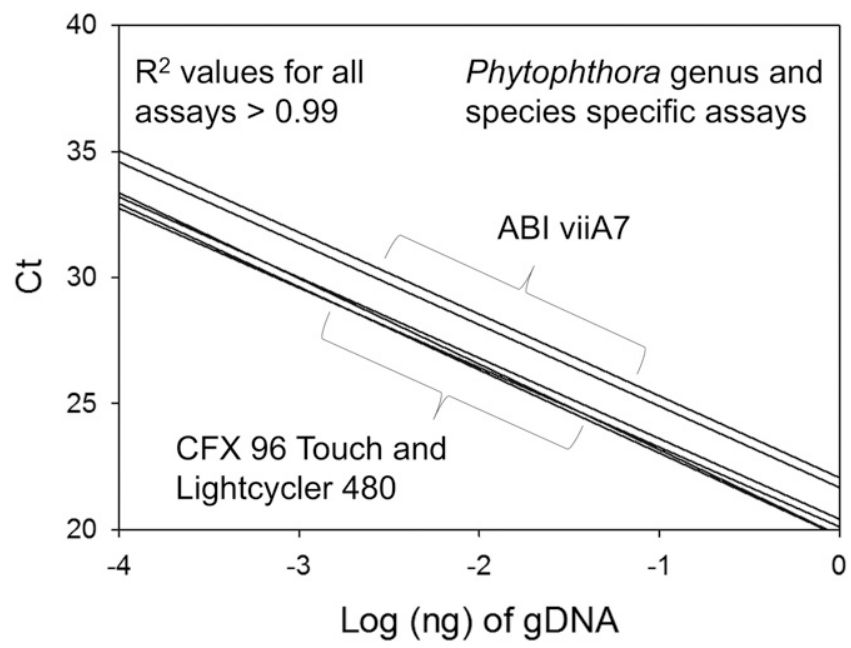

Fig. 3. Technology transfer experiments for the Phytophthora atp9-nad9 detection system comparing a serial dilution of Phytophthora ramorum DNA for genus and species-specific detection. Assays were performed on three separate qPCR machines at two locations.

Table 4. Technology transfer experiments to transfer Phytophthora genus and species-specific TaqMan assays for the atp9-nad9 locus developed in this manuscript. Assays were performed on three different qPCR platforms at two independent locations (USDA-ARS and CFIA). A three step series was conducted including the following steps: Step 1: Confirming amplification of two closely related Phytophthora species (P. fragariae and $P$. rubi). Step 2: Compare sensitivity and slope to the standard (using $P$. ramorum DNA, isolate Prg2). Step 3: Conducting amplifications with blind purified DNA and field samples.

\begin{tabular}{|c|c|c|c|c|}
\hline Step number & Platforms & $\begin{array}{l}0.5 \mathrm{ng} \text { of } P . \text { fragariae } \text { DNA CT } \\
\text { values } \pm \text { SDE (genus/species } \\
\text { detection) }\end{array}$ & $\begin{array}{c}0.5 \mathrm{ng} \text { of } P . \text { rubi DNA CT } \\
\text { values } \pm \mathrm{SDE} \text { (genus/species detection) }\end{array}$ & $\begin{array}{l}0.05 \text { ng of } P \text {. fragariae } \text { spiked into } 5 \mathrm{ng} \text { of } \\
\text { strawberry DNA CT values } \pm \text { SDE } \\
\text { (Phytophthora genus/species/plant detection) }\end{array}$ \\
\hline \multirow{8}{*}{$\begin{array}{l}\text { 1: Positive control samples: } \\
\text { P. fragariae, } P \text {. rubi } \\
\text { and P. fragariae spiked } \\
\text { into strawberry DNA }\end{array}$} & \multirow[t]{2}{*}{ CFX 96 Touch (Bio Rad) ${ }^{\mathbf{a}}$} & $16.46 \pm 0.43 /$ & $17.05 \pm 0.52 /$ & $20.52 \pm 0.36 /$ \\
\hline & & $16.52 \pm 0.52$ & $16.52 \pm 0.52$ & $\begin{array}{l}20.20 \pm 0.72 / \\
22.34 \pm 0.74\end{array}$ \\
\hline & \multirow[t]{2}{*}{ 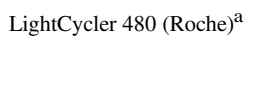 } & $17.42 \pm 0.56 /$ & $18.11 \pm 0.35 /$ & $21.33 \pm 0.42 /$ \\
\hline & & $17.18 \pm 0.47$ & $18.07 \pm 0.42$ & $\begin{array}{l}21.60 \pm 0.64 / \\
\text { Cannot detect }\end{array}$ \\
\hline & \multirow[t]{4}{*}{$\mathrm{ABI}$ viiA7 $(\mathrm{ABI})^{\mathrm{b}}$} & $16.89 \pm 0.65 /$ & $17.63 \pm 0.55 /$ & $20.84 \pm 0.50 /$ \\
\hline & & \multirow[t]{2}{*}{$16.75 \pm 0.52$} & $17.12 \pm 0.87$ & $20.97 \pm 0.68 /$ \\
\hline & & & & $23.01 \pm 0.54$ \\
\hline & & \multicolumn{2}{|c|}{ P. ramorum equations, slope and y intercept (genus/species) } & Limit of detection (genus/species detection limits) \\
\hline \multirow{7}{*}{$\begin{array}{l}\text { 2: Serial dilution: } \\
\text { Purified } P \text {. ramorum DNA }\end{array}$} & \multirow[t]{2}{*}{ CFX 96 Touch (Bio Rad) $)^{\mathrm{a}}$} & \multicolumn{2}{|c|}{$y=-3.295 x+19.739 /$} & $500 \mathrm{fg} /$ \\
\hline & & \multicolumn{2}{|c|}{$y=-3.396 x+19.767$} & $500 \mathrm{fg}$ \\
\hline & \multirow[t]{2}{*}{ LightCycler 480 (Roche) $^{\mathrm{a}}$} & \multicolumn{2}{|c|}{$y=-3.158 x+20.106 /$} & $500 \mathrm{fg} /$ \\
\hline & & \multicolumn{2}{|c|}{$y=-3.194 x+20.411$} & $500 \mathrm{fg}$ \\
\hline & \multirow[t]{3}{*}{$\mathrm{ABI}$ viiA7 $(\mathrm{ABI})^{\mathrm{b}}$} & \multicolumn{2}{|c|}{$y=-3.227 x+21.664 /$} & $500 \mathrm{fg} /$ \\
\hline & & \multicolumn{2}{|c|}{$y=-3.241 x+22.055$} & $500 \mathrm{fg}$ \\
\hline & & $\begin{array}{c}\text { Phytophthora purified DNA } \\
\text { (positive genus level detection out } \\
\text { of total tested/positive species } \\
\text { level detection out of total tested) }\end{array}$ & $\begin{array}{l}\text { Field samples (positive genus level } \\
\text { detection out of total tested/positive } \\
\text { species level detection out of total tested) }\end{array}$ & $\begin{array}{l}\text { Non-Phytophthora purified DNA (positive } \\
\text { genus level detection out of total tested/positive } \\
\text { species level detection out of total tested) }\end{array}$ \\
\hline \multirow{6}{*}{$\begin{array}{l}\text { 3: Blind samples: } 20 \text { samples } \\
\text { (see Table } 3 \text { for more } \\
\text { information) }\end{array}$} & \multirow[t]{2}{*}{ CFX 96 Touch (Bio Rad) ${ }^{a}$} & 5 out of 5 & 4 out of 9 & 0 out of 5 \\
\hline & & 2 out of 5 & 1 out of 9 & 0 out of 5 \\
\hline & \multirow[t]{2}{*}{ 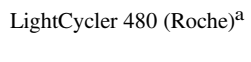 } & 5 out of 5 & 4 out of 9 & 0 out of 5 \\
\hline & & 2 out of 5 & 1 out of 9 & 0 out of 5 \\
\hline & \multirow[t]{2}{*}{$\mathrm{ABI}$ viiA7 $(\mathrm{ABI})^{\mathrm{b}}$} & 5 out of 5 & 4 out of 9 & 0 out of 5 \\
\hline & & 2 out of 5 & 1 out of 9 & 0 out of 5 \\
\hline
\end{tabular}

a Amplifications were performed at the USDA-ARS facility in Salinas, CA.

b Amplifications were performed at the Canadian Food Inspection Agency in Ottawa, ON. 
detection will facilitate diagnostic efforts for this important group of plant pathogens. The previously described assay reported by Bilodeau et al. (2014) addresses this need by using two mitochondrial loci that have a unique gene order in Phytophthora relative to the related genus Pythium, Eumycotan fungi, and plants, thereby reducing the importance of highly controlled annealing temperatures for specificity (two exceptions to this were noted; $P$. bisheria and $P$. frigida had the conserved trnM-trnP-trnM gene order but not the atp9-nad9 gene order). In the initial report, 14 species-specific probes were validated. In this current submission, the validation of an additional 32 speciesspecific probes was completed and supported by a sequence database of the target locus of over 900 isolates representing 145 Phytophthora taxa. A further review of this sequence database supports the systematic nature of marker development for this locus as it should be possible to develop species-specific markers for approximately $89 \%$ of the taxa for which we have sequence data, with probes specific for two or three species possible for a number of the remaining taxa. The validation of species specific TaqMan probes for $P$. sojae and $P$. sansomeana designed from the atp9-nad9 locus was also reported by Rojas et al. (2017).

One problem encountered in the initial report for this technique (Bilodeau et al. 2014) was a reduced amplification efficiency and need for a lower annealing temperature for species such as $P$. nicotianae due to an SNP in the annealing site of the reverse primer. From reviewing the sequence data, this SNP was also observed in $P$. alticola,

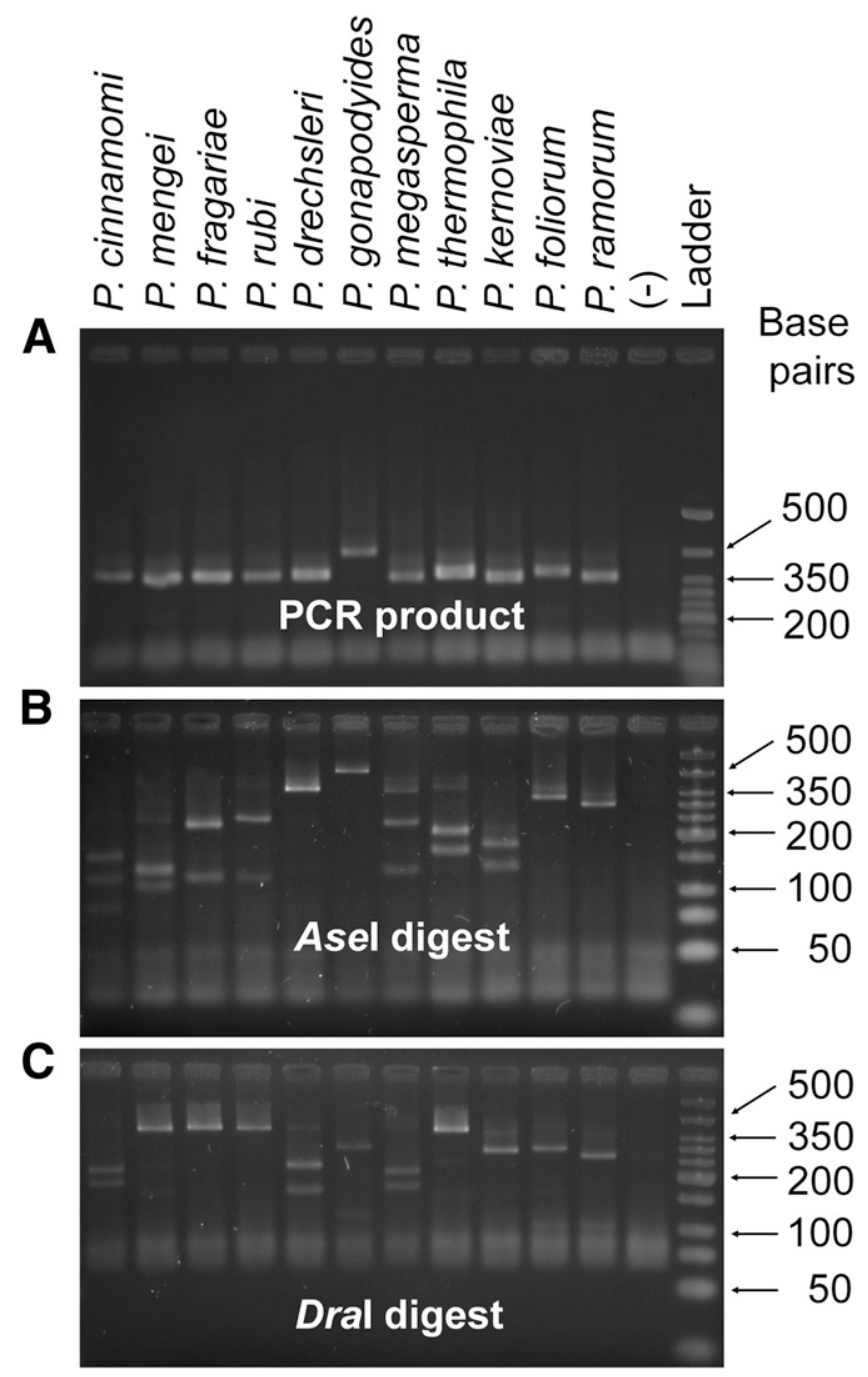

Fig. 4. RFLP analysis of qPCR products from the atp9-nad9 Phytophthora detection system. A, Amplified products from a variety of species visualized on a $1.5 \%$ agarose TBE gel. $\mathbf{B}$ and $\mathbf{C}$ represent these same products following digestion with Asel or Dral, respectively, separated using a 3\% agarose TBE gel.
P. cryptogea (GII), P. elongata, $P$. nicotinae, $P$. medicaginis, $P$. tentaculata, and $P . \times$ pelgrandis. To address this, a second primer containing the SNP was designed (referred to as a "Bandaid" primer) and added to the master mix. When present in a low amount $(8 \mathrm{nM})$, the amplification efficiency was improved and a uniform annealing temperature of $57^{\circ} \mathrm{C}$ could be used for all diagnostic assays. Surprisingly, increasing the added Bandaid primer concentration above $8 \mathrm{nM}$ reduced the sensitivity of detection in several assays. Our hypothesis is that if high concentrations of the bandaid primer persist in the assay, undesired primer dimers may form when template concentrations are low, ultimately disrupting the detection of Phytophthora spp.

When transferring a diagnostic assay from one lab to another, one potential challenge is ensuring consistency in performance, which is especially important when working with quarantine pathogens such as $P$. ramorum. Thermal cyclers may vary in ramping speeds as well as temperature calibration, both of which can have an impact on the specificity and sensitivity of an assay. The complexity of master mix

Table 5. Species that can be identified using species-specific qPCR conventional RFLP using various restriction enzymes (based on in silico predictions) ${ }^{\text {a }}$. Placement of clade was assigned based recent phylogenetic analysis of the genus (Martin et al. 2014)

\begin{tabular}{|c|c|c|c|}
\hline Species & Clade & $\begin{array}{c}\text { Restriction } \\
\text { enzyme(s) required }\end{array}$ & $\begin{array}{l}\text { Other restriction } \\
\text { enzyme options }\end{array}$ \\
\hline austrocedrae & 8 & AseI and DraI & \\
\hline boehmeriae & 10 & AseI and DraI & \\
\hline botryosa & $2 \mathrm{a}$ & AseI and DraI & \\
\hline captiosa & 9 & - & Pst I \\
\hline cinnamomi & $7 b$ & AseI and DraI & \\
\hline drechsleri & $8 \mathrm{a}$ & AseI and DraI & \\
\hline europaea & $7 \mathrm{a}$ & AseI and DraI & \\
\hline foliorum & $8 \mathrm{c}$ & AseI and DraI & \\
\hline gallica & 10 & AseI and DraI & \\
\hline gemini & 6 & AseI and DraI & \\
\hline glovera & $2 b$ & AseI and DraI & \\
\hline gonapodyides & 6 & AseI and DraI & EcoRV \\
\hline hibernalis & $8 \mathrm{c}$ & AseI and DraI & \\
\hline insolita & 9 & AseI and DraI & \\
\hline irrigata & 9 & AseI and DraI & \\
\hline kelmania & $8 \mathrm{a}$ & AseI and DraI & \\
\hline kernoviae & 10 & AseI and DraI & \\
\hline lagoariana & 9 & AseI and DraI & \\
\hline lateralis & $8 \mathrm{c}$ & AseI and DraI & \\
\hline macrochlamydospora $^{\mathrm{c}}$ & 9 & AseI and DraI & \\
\hline medicaginis & $8 \mathrm{a}$ & AseI and DraI & \\
\hline megakarya & 4 & AseI and DraI & \\
\hline megasperma & 6 & AseI and DraI & \\
\hline multivesiculata & 2 & AseI and DraI & \\
\hline multivora & $2 \mathrm{c}$ & AseI and DraI & \\
\hline parvispora & $7 b$ & AseI and DraI & \\
\hline pinifolia & 6 & AseI and DraI & \\
\hline polonica & 9 & AseI and DraI & \\
\hline quercetorum & 4 & AseI and DraI & \\
\hline quercina & 1 & AseI and DraI & \\
\hline quininea $^{\mathrm{c}}$ & 9 & AseI and DraI & \\
\hline ramorum & $8 \mathrm{c}$ & AseI and DraI & XbaI \\
\hline richardiae & 9 & AseI and DraI & \\
\hline robiniae & $7 b$ & AseI and DraI & \\
\hline rubi & $7 \mathrm{a}$ & AseI and DraI & \\
\hline sulawesiensis & 6 & AseI and DraI & \\
\hline tentaculata & $1 b$ & AseI and DraI & \\
\hline
\end{tabular}

a A digestion is considered specific if it can be distinguished from other species using AseI and DraI when a size difference on bands 100 bp or larger differ in size by at least 10 bp or have different banding patterns using both enzymes.

${ }^{\mathrm{b}}$ Other species have specific enzymes that only cut in that particular species. ${ }^{\text {c }} P$. macrochlamydospora and $P$. quininea are closely related and cannot be distinguished from each other but can be distinguished from other Phytophthora spp. using this method. 
setup can also contribute to false negatives due to pipetting errors. To address some of these potential problems, the described Phytophthora assay uses a single primer pair to amplify a locus with a unique gene order in Phytophthora for both genus and species-specific detection, thereby simplifying master mix preparation and reducing potential specificity problems with variation between thermal cyclers. To identify other potential problems with consistency of the assay, trials were run on different thermal cyclers in the authors' labs in California and Ottawa, Canada. While slight differences in amplification efficiency were observed among thermal cyclers, differences in the ability to correctly identify unknown samples were not detected.

One advantage of using a diagnostic assay capable of genusspecific detection is the amplicon can be sequenced to determine what species is present if species-specific detection is negative. While this process is straightforward when only a single species is present, often times multiple species may be encountered when examining environmental samples, leading to a need for amplicon cloning to be able to get clean sequencing reads suitable for species identification by BLAST analysis. In an effort to reduce the need for cloning, a PCR-RFLP procedure with two restriction enzymes was developed that in silico analysis indicated would be effective for this purpose. While not able to differentiate all species due to the limitations of estimating fragment size on an agarose gel, it is possible to identify a number of species due to the uniqueness of the size of digested amplicon fragments. Additional research using techniques to more accurately measure fragment sizes to expand the utility of this approach for species identification from environmental samples are in progress (T. Miles and F. Martin, unpublished). Having a PCR-RFLP system for Phytophthora may be useful for analysis of species communities if the laboratory had limited access to a DNA sequencer.

Due to the size of the atp9-nad9 amplicon and available sequence database (over 900 isolates representing 145 Phytophthora taxa), this locus should also be useful for sequence based analysis of Phytophthora communities, a possibility that is currently being explored (G. Bilodeau, unpublished; F. Martin, unpublished). This database is available currently as an .msf file in Bilodeau et al. (2014) and as a .fasta file in Miles et al. (2015) with an updated alignment as a supplementary file to this submission (Supplementary Alignment). A similar approach has been reported with the ITS region using conventional cloning and sequencing to evaluate the Phytophthora community structure in Scottish streams (Scibetta et al. 2012). Currently the greatest diversity of Phytophthora populations is in irrigation water, which can behave as a reservoir for recombining populations and hybrids (Parke et al. 2014). In a recent study of P. ramorum in Appalachia, oomycetes were isolated on selective media and significant diversity among over 350 isolates was observed. A range of species were identified, including six Phytophthora "Clade 6" species (e.g., $P$. megasperma), members of the $P$. citricola species complex, five unknown Phytophthora species, 11 different Pythium species, three unknown Pythium species, Halophytophthora batemanensis, and one Phytopythium isolate (Shrestha et al. 2013). In another study in South Africa, researchers recovered five novel Phytophthora taxa and many hybrids (Nagel et al. 2013); because of the significant numbers recovered, they are referring to these Phytophthora species as hybrid swarms (Hüberli et al. 2013; Nagel et al. 2013). The atp9nad9 marker system offers an opportunity to study Phytophthora communities in these aquatic ecosystems, which are extremely important to manage their effects on the environment and agriculture.

A number of new Phytophthora spp. have been described, with many identified as invasive to the United States (32 species in total) by the USDA Animal Plant Health Inspection Service and, based on their potential threat to North American agriculture and ecology, were prioritized as their level of risk (Schwartzburg et al. 2009). Another list of off-shore plant pests (Crook 2012) was recently formulated and four Phytophthora spp., as well as other plant pathogenic oomycetes, were listed as being of regulatory concern. The Cooperative Agricultural Pest Survey (CAPS) group develops a pest list every 2 years using an analytical hierarchical process model. The New Pest Advisory Group (NPAG) also provides input to the list looking for new and emerging plant pests (including Phytophthora species).
The focus for development of new species-specific TaqMan probes in this submission was on providing tools for detecting many of these invasive species of regulatory concern in the event that any of them are introduced into North America. With the large number of taxa represented in the in silico database provided in this study, it should be possible to add data from newly described species and rapidly develop/validate a species-specific probe for their detection.

While the TaqMan diagnostic assay described herein is useful in a laboratory setting, the ability to complete diagnostics directly in the field at the point of sample collection would significantly improve the ability to detect invasive species and the chances of preventing introduction. Because of the similarity of primer and probe design to TaqMan, it has been possible to transfer the Phytophthora detection assays to the isothermal detection technology of recombinant polymerase amplification (RPA) (Miles et al. 2015); the trnM-trnPtrnM locus is used for genus-specific and the atp9-nad9 locus for species-specific detection. In an effort to develop a systematic approach for designing species-specific RPA diagnostics, the assays were designed with the probe annealing to the same conserved atp 9 sequences as the TaqMan genus-specific probe with the reverse primer targeting sequences used for the species-specific TaqMan probe. Species-specific detection has been validated for nine species using this approach (Miles et al. 2015; Rojas et al. 2017, T. Miles and F. Martin, unpublished), and given the level of sequence divergence observed in TaqMan probe annealing sites, it is likely that additional species-specific RPA assays can be developed. One significant advantage with the use of the RPA technology is that detection can be observed directly in the field in as little as 15 min of obtaining the plant samples without DNA extraction and using a portable fluorometer with a level of sensitivity that approaches TaqMan real time PCR (Miles et al. 2015).

Molecular diagnostics not only need to be highly specific, but also have the ability to detect the pathogen when it is present in low amounts. To improve sensitivity, marker systems are often designed based on high copy number targets such as the rDNA or the mitochondrial DNA, but if pathogen quantification is also needed, there are several considerations to keep in mind. It has been reported that rDNA may vary in copy number among isolates of Phytopythium vexans (Spies et al. 2011) and this is suggested to also occur in Pythium (Martin 2009; Schroeder et al. 2013); it is unknown if this is a characteristic of Phytophthora spp. Regardless, if quantification is desired when using the described mitochondrial marker system, it is important to keep in mind variation in copy number may be encountered when looking at different structures (zoospore, chlamydospore, oospore), physiological status of the infection, or even at intra- and interspecific levels, so experimental validation of the quantification assay will need to be done.

\section{Acknowledgments}

The authors gratefully acknowledge funding from the USDA - California Department of Food and Agriculture's Specialty Crop Block Grant Program grant SCB12051, the California Avocado Commission, and the USDA-APHIS-PPQCPHST that supported this work. Furthermore, we would like to acknowledge Debbie Shearlaw for assisting in the validation of these tests as well as critical review of the manuscript from Paul Tooley (USDA-ARS).

\section{Literature Cited}

Agler, M. T., Mari, A., Dombrowski, N., Hacquard, S., and Kemen, E. M. 2016. New insights in host-associated microbial diversity with broad and accurate taxonomic resolution. bioRxiv (online), doi:10.1101/050005

Bienapfl, J. C., and Balci, Y. 2014. Movement of Phytophthora spp. in Maryland's nursery trade. Plant Dis. 98:134-144.

Bilodeau, G. J., Coffey, M. D., Blomquist, C., and Martin, F. 2014. Development of a multiplex assay for genus and species specific detection of Phytophthora based on differences in mitochondrial gene order. Phytopathol. 104:733-748.

Crook, S. 2012. The 2012 Prioritized offshore pest list. USDA-APHIS, Riverdale Park, MD.

Drenth, A., Wagels, G., Smith, B., Sendall, B., O’Dwyer, C., Irvine, G., and Irwin, J. 2006. Development of a DNA-based method for detection and identification of Phytophthora species. Australas. Plant Pathol. 35:147-159.

Hüberli, D., Hardy, G. E. S. J., White, D., Williams, N., and Burgess, T. I. 2013. Fishing for Phytophthora from Western Australia's waterways: A distribution and diversity survey. Australas. Plant Pathol. 42:251-260. 
Jung, T., and Burgess, T. I. 2009. Re-evaluation of Phytophthora citricola isolates from multiple woody hosts in Europe and North America reveals a new species, Phytophthora plurivora sp. nov. Persoonia 22:95-110.

Jung, T., Colquhoun, I. J., and Hardy, G. E. J. 2013. New insights into the survival strategy of the invasive soilborne pathogen Phytophthora cinnamomi in different natural ecosystems in Western Australia. For. Pathol. 43:266-288.

Lamour, K., and Hu, J. 2013. Diversity and Phytophthora: A threat to forests, crops and traditional laboratory research - Mini review. CAB Reviews: Perspectives in Agriculture, Veterinary Science, Nutrition and Natural Resources 8. CABI, Wallingford, U.K.

Martin, F. N. 2009. Pythium Genetics. Pages 213-239 in: Oomycete Genetics and Genomics. K. H. Lamour and S. Kamoun, eds. John Wiley and Sons, Hoboken, NJ.

Martin, F. N., Abad, G. Z., Balci, Y., and Ivors, K. 2012. Identification and detection of Phytophthora: reviewing our progress, identifying our needs. Plant Dis. 96:1080-1103.

Martin, F. N., Blair, J. E., and Coffey, M. D. 2014. A combined mitochondrial and nuclear multilocus phylogeny of the genus Phytophthora. Fungal Genet. Biol. 66: 19-32.

Martin, F. N., Tooley, P. W., and Blomquist, C. 2004. Molecular detection of Phytophthora ramorum, the causal agent of sudden oak death in California, and two additional species commonly recovered from diseased plant material. Phytopathology 94:621-631.

Mendes, L. W., Kuramae, E. E., Navarrete, A. A., Van Veen, J. A., and Tsai, S. M. 2014. Taxonomical and functional microbial community selection in soybean rhizosphere. ISME J. 8:1577-1587.

Miles, T. D., Martin, F. N., and Coffey, M. D. 2015. Development of rapid isothermal amplification assays for detection of Phytophthora species in plant tissue. Phytopathology 105:265-278.

Nagel, J. H., Gryzenhout, M., Slippers, B., Wingfield, M. J., Hardy, G. E., Stukely, M. J. C., and Burgess, T. I. 2013. Characterization of Phytophthora hybrids from ITS clade 6 associated with riparian ecosystems in South Africa and Australia. Fungal Biol. 117:329-347.

Parke, J. L., Knaus, B. J., Fieland, V. J., Lewis, C., and Grünwald, N. J. 2014 Phytophthora community structure analyses in Oregon nurseries inform systems approaches to disease management. Phytopathology 104:1052-1062.

Rojas, J. A., Miles T. D., Coffey, M. D., Martin, F. N., and Chilvers, M. 2017 Development and application of qPCR and RPA genus and species-specific detection of Phytophthora sojae and Phytophthora sansomeana root rot pathogens of soybean. Plant Dis. (in press) doi:10.1094/PDIS-09-16-1225-RE

Schena, L., Duncan, J., and Cooke, D. 2008. Development and application of a PCR-based 'molecular tool box' for the identification of Phytophthora species damaging forests and natural ecosystems. Plant Pathol. 57:64-75.

Schroeder, K. L., Martin, F. N., de Cock, A. W., Lévesque, C. A., Spies, C. F. Okubara, P. A., and Paulitz, T. C. 2013. Molecular detection and quantification of Pythium species: evolving taxonomy, new tools, and challenges. Plant Dis. 97:4-20.

Schwartzburg, K., Hartzog, H., Landry, C., Rogers, J., and Randall-Schadel, B. 2009. Prioritization of Phytophthora of concern to United States. USDA APHIS PPQ CPHST PERAL (Plant Epidemiology and Risk Analysis Laboratory), Raleigh, NC.

Scibetta, S., Schena, L., Chimento, A., Cacciola, S. O., and Cooke, D. E. 2012. A molecular method to assess Phytophthora diversity in environmental samples. J. Microbiol. Methods 88:356-368.

Shrestha, S. K., Zhou, Y., and Lamour, K. 2013. Oomycetes baited from streams in Tennessee 2010-2012. Mycologia 105:1516-1523.

Spies, C. F., Mazzola, M., Botha, W. J., Van Der Rijst, M., Mostert, L., and Mcleod, A. 2011. Oogonial biometry and phylogenetic analyses of the Pythium vexans species group from woody agricultural hosts in South Africa reveal distinct groups within this taxon. Fungal Biol. 115:157-168. 